\title{
Review
}

\section{Parkinson's Disease Drug Development Since 1999: A Story of Repurposing and Relative Success}

\author{
Deirdre M. Boucherie ${ }^{\mathrm{a}, \mathrm{b}, 1}$, Gonçalo S. Duarte ${ }^{\mathrm{b}, \mathrm{c}, \mathrm{d}, 1}$, Tiago Machado ${ }^{\mathrm{b}, \mathrm{c}}$, Patrícia R. Faustino ${ }^{\mathrm{b}, \mathrm{c}}$, \\ Cristina Sampaio $^{\mathrm{c}, \mathrm{e}}$, Olivier Rascol ${ }^{\mathrm{f}}$ and Joaquim J. Ferreira ${ }^{\mathrm{b}, \mathrm{c}, \mathrm{g}, *}$ \\ ${ }^{\text {a }}$ Swammerdam Institute for Life Sciences, Center for Neuroscience, University of Amsterdam, Amsterdam, \\ The Netherlands \\ ${ }^{\mathrm{b}}$ Laboratory of Clinical Pharmacology and Therapeutics, Faculdade de Medicina, Universidade de Lisboa, \\ Lisboa, Portugal \\ ${ }^{\mathrm{c}}$ Instituto de Medicina Molecular, Faculdade de Medicina, Universidade de Lisboa, Lisboa, Portugal \\ ${ }^{\mathrm{d}}$ Centro de Estudos de Medicina Baseada na Evidência, Faculdade de Medicina, Universidade de Lisboa, Lisboa, \\ Portugal \\ ${ }^{\mathrm{e}}$ CHDI Management/CHDI Foundation, Princeton, NJ, USA \\ ${ }^{\mathrm{f}}$ Department of Clinical Pharmacology and Neurosciences, Centre d'Investigation Clinique CIC1436, NS- \\ Park/FCRIN Network, UMR ToNIC 1214, University Hospital of Toulouse, INSERM, University of Toulouse \\ 3, Toulouse, France \\ ${ }^{\mathrm{g}}$ CNS - Campus Neurológico Sénior, Torres Vedras, Portugal
}

Accepted 6 December 2020

Pre-press 11 January 2021

\begin{abstract}
.
Background: A global overview of drug development programs in Parkinson's disease over the last few decades is lacking, while such programs are challenging given the multifaceted and heterogeneous nature of the disease.

Objective: To indirectly assess drug development programs in Parkinson's disease, exploring some factors associated with compound attrition at different trial phases.

Methods: We assessed all Parkinson's disease trials in the WHO trials portal, from inception (1999) to September 2019. Independent authors selected trials and extracted data. The success rate was the number of compounds that progressed to the next drug development phase divided by the number of compounds in that phase.

Results: Overall, 357 trials (studying 152 compounds) fulfilled our inclusion criteria, with 62 (17.3\%) phase 1 trials, 135 (37.8\%) phase 2 trials, $85(23.8 \%)$ phase 3 trials, and 53 (14.8\%) phase 4 trials. The success rate was $42.4 \%$ from phase 2 to 3 . Original compounds received regulatory approval by the FDA in $21.4 \%$ of cases, compared with $6.7 \%$ of repurposed compounds, representing an overall success rate of $14.9 \%$. We found 172 trials $(48.2 \%)$ conducted for repurposing previously licensed compounds. These figures were approximately the same regarding approval by the EMA. Most compounds were approved to treat parkinsonism and motor fluctuations.
\end{abstract}

\footnotetext{
${ }^{1}$ These authors contributed equally to this study.

*Correspondence to: Joaquim J. Ferreira, MD, PhD, Avenida Prof. Egas Moniz, 1649-028, Lisboa, Portugal. E-mail: jferreira@ medicina.ulisboa.pt.
} 
Conclusion: We found a moderate-to-high success rate in all phases of drug development. This was largely based on the success of original compounds, despite almost half of the identified trials attempting compound repurposing.

Keywords: Clinical development, drug development, clinical trials, parkinson's disease

\section{INTRODUCTION}

Parkinson's disease is the second most common age-related neurodegenerative disorder after Alzheimer's disease [1].

Levodopa continues to be the most efficacious therapeutic drug for Parkinson's disease treatment [2], though as Parkinson's disease is a multifaceted condition, additional elements, such as dementia, falls, drug-induced dyskinesia, among others, mean that the treatment of Parkinson's disease is not entirely satisfactory. The number of compounds for treating Parkinson's disease continues to expand, though the number of licensed compounds in the past 10 years has been sparse [3].

The World Health Organization (WHO) International Clinical Trials Search Portal (ICTRP) contains the trial registration datasets provided by 17 clinical trial registries [4]. Clinicaltrials.gov was made publicly available in 2000, and is the largest clinical trials registry, representing about $75 \%$ of available trials in WHO ICTRP. Since 2005, the International Committee of Medical Journal Editors (ICMJE) requires trial registration as a condition for publication [5]. In 2006, Food and Drug Administration (FDA) stated that all trials should be registered [6]. Currently, it is anticipated that the great majority of ongoing clinical trials are, or will be, captured and recorded by clinical trials databases [7].

We examined the ICTRP to determine the characteristics of Parkinson's disease trials registered since its inception in 1999, including currently ongoing trials. We aimed to assess the history of drug development in Parkinson's disease, characterize trends in drug development, and assess characteristics of candidate therapies. Ultimately, we want to provide a snapshot of drug development in Parkinson's disease.

\section{METHODS}

We searched the ICTRP for interventional trial records from inception (1999) to September 2019 using the term "Parkinson". We included only trials of pharmacological compounds. We combined the abstracted data available in the original records of the clinical trials registries. We extracted the following data onto a pre-piloted spreadsheet: trial title, phase, Anatomical Therapeutic Chemical (ATC) classification categories, sponsor, funder, registration and last updated dates, trial identification number, status, publication status in completed trials, reason for failure, major safety issue identified, results available, study start and study estimated end dates, study duration, participant numbers, location, inclusion and exclusion criteria, primary study aim based on the Movement Disorders Society Evidence-Based Medicine [8, 9] (disease-modifying, early Parkinson's disease requiring treatment, motor fluctuations, parkinsonism, dementia associated with Parkinson's disease, psychosis associated with Parkinson's disease, orthostatic hypotension associated with Parkinson's disease, depression associated with Parkinson's disease, falls, sleep disorders, other neuropsychiatric), randomization method, control group, primary and secondary endpoints, endpoint classification, study design, blinding, primary endpoint, and biomarkers reported. Biomarkers were defined as a characteristic that is objectively measured and evaluated as an indicator of normal biologic processes, pathogenic processes, or pharmacologic responses to an intervention [10]. The primary study aim 'early Parkinson's disease requiring treatment' was used when stated as such in the registry and when these trials concerned the whole spectrum of both motor and non-motor symptoms, including the aims of slowing down disease progression and delaying the start of levodopa treatment.

Trials were also classified into those assessing a potential disease modifying effect or those assessing a symptomatic effect, according to the main objective of the primary endpoint. We classified repurposing trials as the utilization of a known compound, licensed by FDA or EMA, in a novel indication, underscoring a new mode of action that predicts innovative therapeutic options [11]. We distinguished compounds with a known antiparkinsonian effect but with approval for different aspects of Parkinson's than the studied treatment indication, and compounds approved for indications outside Parkinson. Lastly, we assessed whether compounds were approved by 


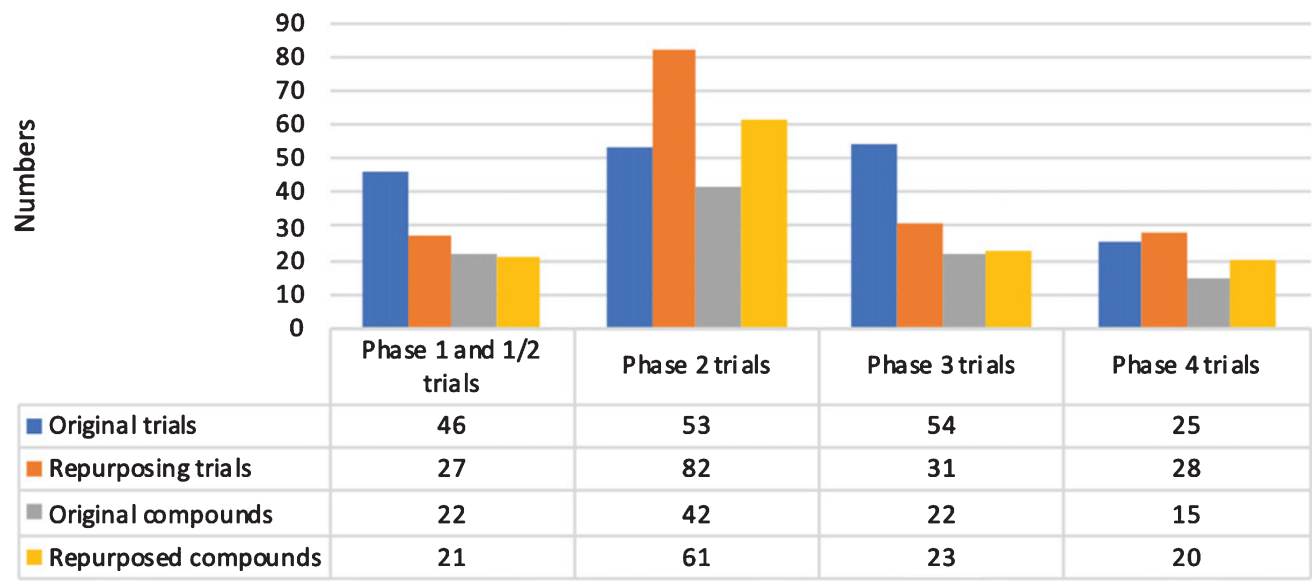

Fig. 1. Overview of trials and compounds by phase.

the European Medicines Agency (EMA) and FDA for the studied treatment indication.

Independent authors selected the trials and extracted data. We excluded trials on nonpharmacological interventions, including behavior interventions, surgical interventions, stem cell therapies, herbs, dietary supplements, and probiotics. We did not exclude trials due to incomplete data reporting, though we searched for publications if data was missing from the online registries.

Disease stages were extracted from data available in the original records or clinical trials. For this study the term early PD was used when original records used this definition. Moderate PD was used as term when original records stated that subjects were in a further stage than early PD, but did not meet requirements for the definition of advanced Parkinson's disease. We considered advanced Parkinson's disease as one of: dyskinesia, motor fluctuations, on/off phenomena, or wearing off; we considered late-stage Parkinson's disease as the presence of one of: autonomic dysfunction, dementia, falls, orthostatic hypotension, or psychosis. When patients of any disease stage were accepted as subjects, or when nothing about the disease stage was defined in the trial data, we used the term all disease stages. When multiple disease stages were studied in a trial, we named all applicable disease stages. We accepted the definitions of trial phases and indications displayed on the trial registries.

We conducted statistical analyses using the Statistical Package of Social Sciences version 20.0.0 (SPSS Inc., Chicago, IL, USA). We calculated descriptive statistics, namely one-way analysis of variance (ANOVA) with Tukey's post hoc test for comparisons of continuous variables of more than two groups. We deemed $P$ values of 0.05 statistically significant, with tests being two-sided. We calculated success rates for each trial phase, which was calculated as the number of compounds that progressed to the next trial phase divided by the number of compounds in that phase. The overall success rate was calculated as the ratio between the number of approved compounds and the total number of compounds from all phases.

\section{RESULTS}

\section{Overview}

Our search identified 1580 references from 1999 to September 2019 and we included 357 trials, assessing 152 compounds. Notably, we excluded 720 trials that used a non-pharmacological intervention and 392 observational studies, $58.4 \%$ and $31.8 \%$ of the excluded trials, respectively. Clinicaltrials.gov provided the data for 251 trials $(70.3 \%)$. The number of registered trials was highest in 2017 (44, 12.3\%), with only 17 being registered before 2004. See Supplementary Material for more details.

We included $62(17.3 \%)$ phase 1 trials, $11(3.1 \%)$ phase $1 / 2$ trials, 135 (37.8\%) phase 2 trials, 85 (23.8\%) phase 3 trials, $53(14.8 \%)$ phase 4 trials, and $11(3.1 \%)$ trials with a non-specified phase (Fig. 1). Overall, 203 trials (56.8\%) were classified as completed, $114(31.9 \%)$ as ongoing, 29 as prematurely terminated $(8.1 \%)$, and $11(3.1 \%)$ as of unknown status. We found 172 trials $(48.2 \%)$ that were aimed at repurposing previously approved compounds for a new indication. 
Table 1

Publication frequency of completed trials

\begin{tabular}{lccc}
\hline Phase & $\begin{array}{c}\text { Completed } \\
\text { trials }\end{array}$ & $\begin{array}{c}\text { Completed trials } \\
\text { with publication }\end{array}$ & Percentage \\
\hline 1 & 47 & 14 & $29.8 \%$ \\
$1 / 2$ & 3 & 1 & $33.3 \%$ \\
2 & 90 & 26 & $28.9 \%$ \\
3 & 64 & 28 & $43.8 \%$ \\
4 & 33 & 16 & $48.5 \%$ \\
Not specified & 7 & 1 & $14.3 \%$ \\
Total & 244 & 86 & $35.2 \%$ \\
\hline
\end{tabular}

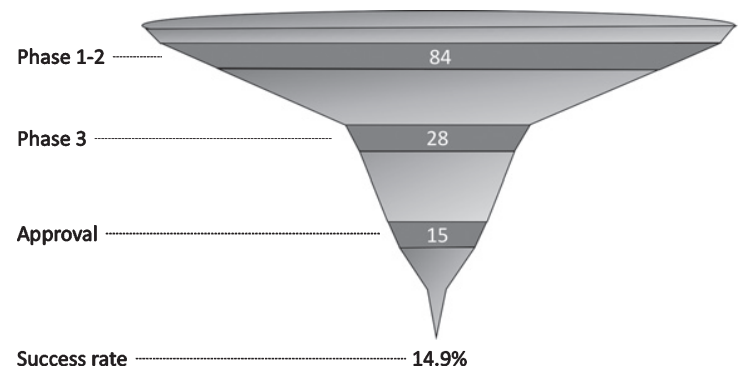

Fig. 2. Number of compounds by phase and overall success rate since 1999.

Most trials were conducted in patients with advanced Parkinson's disease $(83,23.2 \%)$, early to advanced Parkinson's disease $(52,14.6 \%)$, and in all disease stages $(51,14.3 \%)$. See Supplementary Material for more details on disease stages.

There was a significant difference in study duration $(p<0.001)$, treatment duration $(p<0.001)$ and number of participants $(p<0.001)$ between phases.

Of 244 completed trials, $86(35.2 \%)$ were published, the most in phases 3 (28 of 64 trials, $43.8 \%$ ) and 4 (16 of 33 trials, $48.5 \%$; see Table 1 ).

\section{Regulatory approval}

The success rate between phases 1 and 2 was not calculated as the number of compounds was larger in phase 2. The success rate between phases 2 and 3 was $42.4 \%$. We found 14 EMA-approved compounds and 15 FDA-approved compounds, representing an overall success rate of $13.9 \%$ and $14.9 \%$, respectively (Fig. 2).

Only compounds aimed at treatment of parkinsonism and motor fluctuations received EMA approval. The FDA additionally approved compounds aimed at the treatment of dyskinesia, orthostatic hypotension, and psychosis associated with Parkinson's disease.

Regarding EMA approval, 12 of 56 original compounds were approved (21.4\%), compared to two of
45 repurposed compounds (4.4\%). Regarding FDA approval, 12 of 56 original compounds were approved (21.4\%), compared to three of 45 repurposed compounds $(6.7 \%)$.

Among completed and repurposed trials, six of 52 compounds $(11.5 \%)$ had a known antiparkinsonian effect. The repurposed compounds and their original regulatory indication can be seen in the Supplementary Material.

\section{Phase 1 trials}

We identified 62 phase 1 and 11 phase $1 / 2$ trials, studying 38 and 10 compounds, respectively (see Supplementary Material). Overall, 22 phase 1 trials $(35.5 \%)$ and six phase $1 / 2$ trials $(54.5 \%)$ were aimed at repurposing. The majority of phase 1 trials were conducted in healthy subjects $(31,50.0 \%)$. The most common treatment indications of phase 1 trials were motor fluctuations (28 trials, $45.1 \%$ ) and parkinsonism (15 trials, $24.2 \%$ ).

The most frequently identified therapeutic categories were antiparkinsonian drugs (30 trials), and compounds that did not have an ATC therapeutic category yet ( 23 trials).

The most commonly identified compounds were opicapone (18 trials), levodopa in different formulations and trans-resveratrol (both six trials), PBF-509, ropinirole, and rotigotine (all two trials).

The median number of participants in phase 1 trials was 25 (interquartile range [IQR]: 18.25-40.75), the median study duration was 289 days (IQR: 91-509.5), and the median treatment duration was three days (IQR: 1-10, one trial missing). The median number of participants in phase $1 / 2$ trials was 26 (IQR: 20-37.5, one trial missing), the median study duration was 365 days (IQR: 167.5-511.5), and the median treatment duration was 25 days (IQR: 7.5-70, one trial missing).

\section{Phase 2 trials}

We identified 135 phase 2 trials, studying 99 compounds (Fig. 3). Overall, 86 trials (63.7\%) were aimed at repurposing. Most trials were conducted in patients with advanced Parkinson's disease $(29,21.5 \%)$, early to advanced Parkinson's disease $(23,17.0 \%)$, and in all disease stages $(21,15.6 \%)$. The most common treatment indications of phase 2 trials were parkinsonism (28 trials, 20.7\%) and motor fluctuations (22 trials, $16.3 \%)$. 


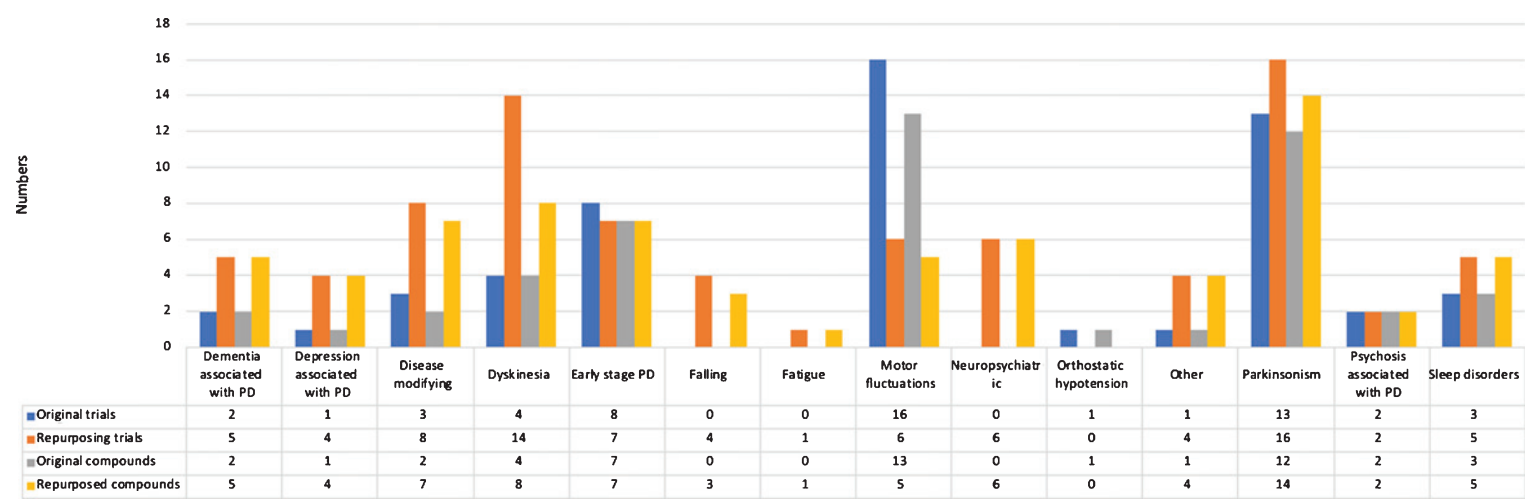

Fig. 3. Overview of trials and compounds in phase 2 trials.

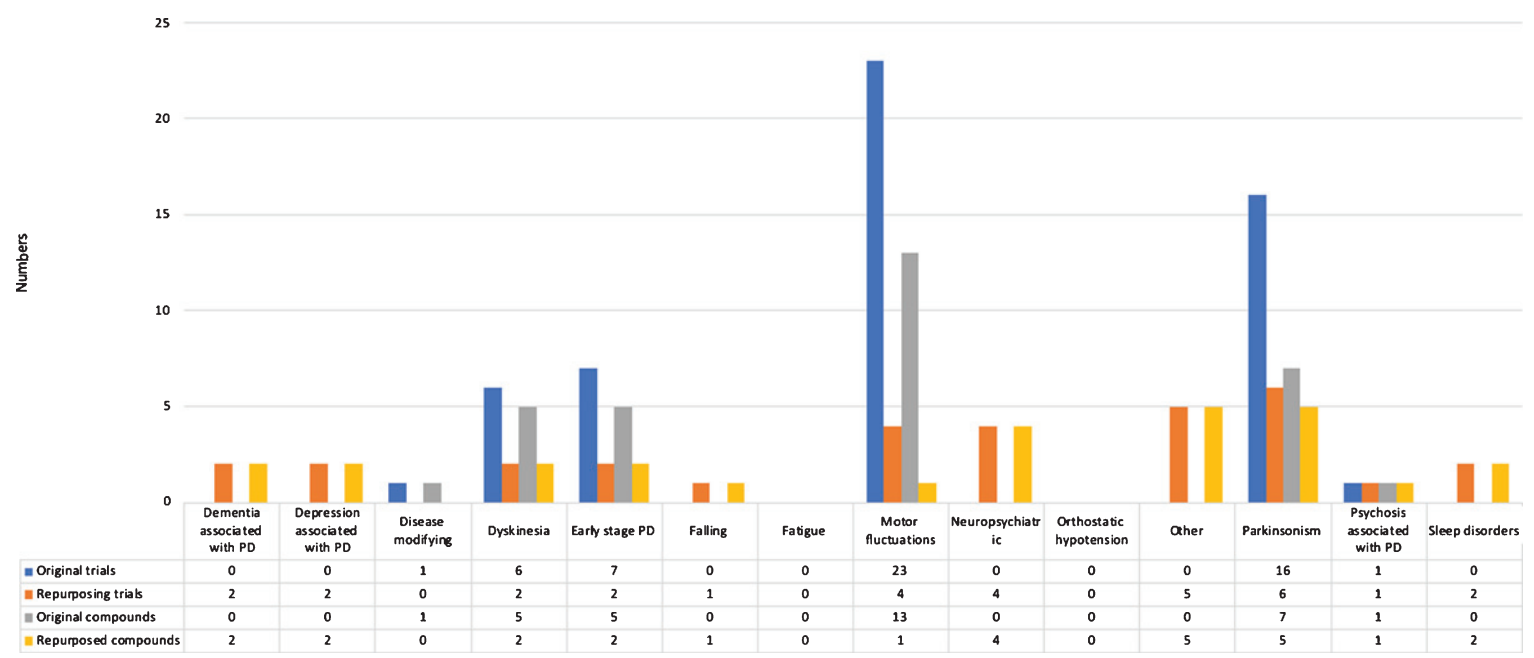

Fig. 4. Overview of trials and compounds in phase 3 trials.

The most frequently identified therapeutic categories were compounds without ATC therapeutic category (37 trials), antiparkinsonian drugs ( 24 trials), and psychoanaleptics (12 trials).

The most commonly identified compounds were levodopa in different formulations (seven trials), safinamide (four trials), and amantadine, botulinum neurotoxin type A, donepezil, droxidopa, mavoglurant, nicotine, paliroden, preladenant (all three trials).

The median number of participants was 42 (IQR: 20-94, one trial missing), the median study duration was 584 days (IQR: 365-974, 12 trials missing), and the median treatment duration was 84 days (IQR: 29-182.6, 19 trials missing).

\section{Phase 3 trials}

We identified 85 phase 3 trials, studying 43 compounds (Fig. 4). Overall, 35 trials (41.2\%) were aimed at repurposing. Most trials were conducted in patients with advanced Parkinson's disease (27, $31.8 \%$ ), early Parkinson's disease $(16,18.8 \%)$, and all disease stages $(12,14.1 \%)$. The most common treatment indications of phase 3 trials were motor fluctuations (27 trials, 31.8\%) and parkinsonism (22 trials, $25.9 \%$ ).

The most frequently identified therapeutic categories were antiparkinsonian drugs (39 trials), compounds without ATC therapeutic category (26 trials), and psychoanaleptics (nine trials).

The most commonly identified compounds were ropinirole (10 trials), pramipexole (nine trials), and levodopa in different formulations (six trials).

The median number of participants was 219 (IQR: 76-446), the median study duration was 730 days (IQR: 487-1126, three trials missing), and the median treatment duration was 168 days (IQR: 84-231, 12 trials missing). 


\section{Phase 4 trials}

We identified 53 phase 4 trials, studying 28 compounds (see Supplementary Material). Overall, 28 trials $(52.8 \%)$ were aimed at repurposing. Most trials were conducted in patients with advanced Parkinson's disease $(15,28.3 \%)$, early to advanced Parkinson's disease $(13,24.5 \%)$, and all disease stages $(11,20.8 \%)$. The most common treatment indications of phase 4 trials were motor fluctuations ( 25 trials, 30.8\%) and parkinsonism (22 trials, 27.2\%).

The most frequently identified therapeutic categories were antiparkinsonian drugs (31 trials) and psychoanaleptics (seven trials).

The most commonly identified compounds were levodopa in different formulations (eight trials), pramipexole (seven trials), and ropinirole (six trials).

The median number of participants was 95 (IQR: 32-535), the median study duration was 822 days (IQR: 548-273.9, two trials missing), and the median treatment duration was 84 days (IQR: 61-365, nine trials missing).

\section{Current drug pipeline}

Among the 114 ongoing trials, the majority (46, $40.4 \%$ ) are phase 2 (See Supplementary Material). Most trials are aimed at repurposing (78, 68.4\%). Most trials are being conducted in patients with early-to-advanced stage $(32,28.1 \%)$, advanced stage $(17,14.9 \%)$, and early stage Parkinson's disease (17, $14.9 \%$ ). The most common treatment indications of ongoing trials are parkinsonism (22 trials, 18.3\%), motor fluctuations (14 trials, $12.3 \%$ ), and disease modification (11 trials, $9.6 \%$ ).

The most frequently identified therapeutic categories are compounds without ATC therapeutic category (29 trials), antiparkinsonian drugs (26 trials), and psychoanaleptics (10 trials).

The most commonly identified compounds are levodopa in different formulations (12 trials), droxidopa, safinamide, and rasagiline (all five trials), and amantadine (four trials).

Of ongoing trials, 12 of 56 compounds have a known antiparkinsonian effect $(21.4 \%)$.

\section{Trial location}

Most trials were conducted exclusively in Europe (116 trials, $32.5 \%$ ) or the USA (91 trials, $25.5 \%$ ), while 79 trials $(22.1 \%)$ were conducted exclusively in other countries (see Supplementary Material).

\section{Trial funding}

Most trials were industry-funded: $202(56.6 \%)$ trials exclusively industry-funded and $44(12.3 \%)$ both by industry and other entities. The remaining 111 trials $(31.1 \%)$ were funded by universities, hospitals, or other. Of trials repurposing compounds, many were industry-funded: $56(32.6 \%)$ trials exclusively industry-funded and $30(17.4 \%)$ both by industry and other entities (see Supplementary Material).

\section{Biomarkers}

Overall, 48 trials reported biomarkers, 35 being phase 2 trials $(25.9 \%)$ and 13 being phase 3 trials $(15.3 \%)$. The most commonly used biomarkers were plasma drug concentrations (20 trials), plasma target engagement and DaT-SPECT (both eight trials), and CSF drug concentrations (six trials). See Supplementary Material for more information.

\section{Disease modifying trials}

We classified 47 trials (13.2\%) as assessing a potential disease modifying effect of 38 different compounds. $26(55.3 \%)$ trials are aimed at repurposing. Most trials are phase $1(14,29.8 \%)$ or phase 2 trials $(25,53.2 \%)$. Most trials included early stage PD patients: 25 recruiting exclusively early stage patients and 16 recruiting patients in other stages as well.

Disease modifying trials have longer durations than those not classified as such (730 vs 548 days of median duration, $p<0.01$ ).

\section{DISCUSSION}

We aimed to approach indicators of drug development programs in Parkinson's disease by using the data available in the ICTRP, accessible since 1999.

We found a relatively high success rate of $14.9 \%$, compared with 3.5\% for Huntington disease [12], 9\% for neurological diseases in general [13], and up to around $19 \%$ for cancer therapies [14]. This success rate for Parkinson's disease likely serves as an incentive for greater investment on the part of industry, thereby potentially increasing the likelihood of future developments.

Despite the relatively high success rate, most compounds remain, regarding regulatory approval, failures. The high attrition rate is difficult to study retrospectively given the lack of information in the ICTRP or in publications regarding the reasons 
behind the failures. Given the large placebo and nocebo response in Parkinson's disease, questions of relative efficacy and safety are particularly important, these being at least partially dependent on trial characteristics [15].

In drug investigation, especially when so many compounds are repurposed, these may not necessarily go through each development phase. This non-linearity may be a contributor to the small number of phase 1 trials, and the high overall success rate. The paucity of phase 1 trial data is likely also due to the fact that these are not always required to be registered under EU and USA regulations [16, 17]. Moreover, there is evidence of a greater publication bias regarding phase 1 trials when compared with other trial phases [18], which may bias our estimates.

The trial characteristics that differ between trial phases are the standard differences in condition characteristics (healthy volunteers in phase 1 trials), participant enrolment (most in phase 3 trials), and trial duration (greatest in phase 3 trials).

The very small number of trials registered before 2004 likely does not reflect the true number of trials during that period, since major policies on trial registration, such as the International Committee of Medical Journal Editors statement [19] and the FDA Amendments Act [16] were only implemented in 2005 and 2007 respectively, which may raise some doubts concerning the data on this period.

The success rate is largely based on the successes of original compounds, with few approvals being granted for repurposed compounds by either the EMA or FDA. The lack of success among repurposed compounds is at odds with the very large number of ongoing trials based on repurposed compounds. The approved original compounds are all related to the dopaminergic system and approved for motor manifestations. The approved repurposed compounds are also related to the dopaminergic system, except for the norepinephrine precursor droxidopa which is FDA-approved for orthostatic hypotension. The outcomes of the trials assessing repurposed compounds are not recorded in the registries, making it difficult to assess reasons of why these compounds were not approved.

Despite the multifaceted and heterogeneous nature of Parkinson's disease, compounds have mainly been approved to treat only parkinsonism and motor fluctuations. The reasons behind this limited success in the non-motor manifestations of Parkinson's disease may be related to, among other factors, sparse preclinical data, the lack of validated animal models, and the inexistence of recommendations by the EMA or FDA regarding this issue. The success of compounds treating motor manifestations may be due to an anchoring effect around the established motor benefit of levodopa. This is at odds with the fact that the market for non-motor manifestations is likely large, as these manifestations are integral in advanced disease states, and so would constitute considerable commercial interest.

Biomarkers used in past trials do not give adequate information regarding disease progression. The small number of trials that report biomarkers is also of interest, as if more data were available it would be possible, even when trials fail to meet their primary aims, to learn about the mechanisms that may be behind the failure.

Until now, no disease-modifying treatments have been approved. Due to the lack of progression biomarkers and the heterogeneous pathophysiology, a change in disease progression cannot yet be measured easily [20-22]. We need to look further than clinical and imaging assessments and need to develop tools more apt to identify small but significant changes in disease progression. Moreover, advanced therapies such as those targeting genetic causes might be of interest to follow in the future.

Most trials are industry-funded, with the proportion $(68.9 \%)$ being greater than the corresponding proportion in other neurological conditions such as Alzheimer disease (56.6\%) [23] and Huntington disease $(60.6 \%)$ [12].

Ongoing trials have approximately the same proportion of repurposed compounds with a known antiparkinsonian effect as completed trials. This leads us to hypothesize that the trial successes will likely decrease, owing to the increasing number of repurposed compounds identified, as these historically have not received regulatory approval.

This study has several limitations. First, the data presented focus exclusively on registered trials. Similarly, as previously observed, phase 1 trials may be underrepresented. Second, there is a significant amount of missing or unsubmitted data for certain data fields, which limits the completeness of the analyses and thus the interpretability of the results presented. Third, our results may not contemplate all ongoing trials, an important issue given the common nature of retrospective registration [24]. Fourth, trials were conducted all over the world, though, for simplicity, we only studied regulatory approval according to the EMA and FDA. Finally, assessing the preclinical pipeline was outside the scope of the current 
review but would be helpful for looking into future drug development.

Although techniques such as gene therapy, stem cell treatments, fetal microbiota transplantations, and deep-brain stimulation (DBS) were outside the scope of the current review, assessing these treatments would provide a more complete landscape of treatment options. Currently approved treatments such as DBS play a large role in managing Parkinson's disease, and even other conditions [24, 25].

In conclusion, the analysis of the trajectory of clinical trials provides significant resources for improving future drug development. Even though it is difficult to monitor trends in Parkinson's disease trials over time, we show that it is still feasible. A large attrition rate for repurposed compounds, a relative success for original compounds, and the exclusive approval of compounds in a narrow range of symptomatic treatment for Parkinson's disease were observed. The overall success rate was $14.9 \%$, corresponding to 15 compounds approved by the FDA since 1999. Still, 114 trials are being conducted, testing 100 different compounds, though the course of these trials remains to be seen.

\section{CONFLICT OF INTEREST/FINANCIAL DISCLOSURES}

Joaquim J Ferreira: received speaker and consultant fees from GlaxoSmithKline, Novartis, TEVA, Lundbeck, Solvay, Abbott, Bial, Merck-Serono, Grunenthal, and Merck Sharp and Dohme.

Olivier Rascol: received fees for scientific advising from AbbVie, Adamas, Acorda, Addex, Apopharma, Bial, Biogen, Britannia, Clevexel, Cynapsus, INC Reasearch, Lundbeck, Merck, MundiPharma, Neuroderm, Novartis, Oxford Biomedica, Parexel, Pfizer, and Prexton.

Cristina Sampaio, Deirdre M. Boucherie, Gonçalo S. Duarte, Patrícia R. Faustino, Tiago Machado: None.

\section{SUPPLEMENTARY MATERIAL}

The supplementary material is available in the electronic version of this article: http://dx.doi.org/ 10.3233/JPD-202184.

\section{REFERENCES}

[1] Dorsey ER, Constantinescu R, Thompson JP, Biglan KM, Holloway RG, Kieburtz K, Marshall FJ, Ravina BM, Schi- fitto G, Siderowf A, Tanner CM (2007) Projected number of people with Parkinson disease in the most populous nations, 2005 through 2030. Neurology 68, 384.

[2] LeWitt PA (2015) Levodopa therapy for Parkinson's disease: Pharmacokinetics and pharmacodynamics. Mov Disord 30, 64-72.

[3] Fox SH, Katzenschlager R, Lim S-Y, Barton B, de Bie RMA, Seppi K, Coelho M, Sampaio C, on behalf of the Movement Disorder Society Evidence-Based Medicine Committee (2018) International Parkinson and movement disorder society evidence-based medicine review: Update on treatments for the motor symptoms of Parkinson's disease. Mov Disord 33, 1248-1266.

[4] Ogino D, Takahashi K, Sato H (2014) Characteristics of clinical trial websites: Information distribution between ClinicalTrials.gov and 13 primary registries in the WHO registry network. Trials $\mathbf{1 5}, 428$.

[5] Zarin DA, Tse T, Williams RJ, Califf RM, Ide NC (2011) The ClinicalTrials.gov results database-update and key issues. N Engl J Med 364, 852-860.

[6] U.S National Library of Medicine (2007) Food and Drug Administration Amendments Act of 2007, Public Law No. 110-85 $§ 801$. Food and Drug Administration.

[7] Harriman SL, Patel J (2016) When are clinical trials registered? An analysis of prospective versus retrospective registration. Trials 17, 187.

[8] Fox SH, Katzenschlager R, Lim SY, Barton B, de Bie RMA, Seppi K, Coelho M, Sampaio C; Movement Disorder Society Evidence-Based Medicine Committee (2018) International Parkinson and movement disorder society evidence-based medicine review: Update on treatments for the motor symptoms of Parkinson's disease. Mov Disord 33, 1248-1266.

[9] Seppi K, Ray Chaudhuri K, Coelho M, Fox SH, Katzenschlager R, Perez Lloret S, Weintraub D, Sampaio C; the collaborators of the Parkinson's Disease Update on NonMotor Symptoms Study Group on behalf of the Movement Disorders Society Evidence-Based Medicine Committee (2019) Update on treatments for nonmotor symptoms of Parkinson's disease-an evidence-based medicine review. Mov Disord 34, 180-198.

[10] Frank R, Hargreaves R (2003) Clinical biomarkers in drug discovery and development. Nat Rev Drug Discov 2, 566580.

[11] Langedijk J, Mantel-Teeuwisse AK, Slijkerman DS, Schutjens MH (2015) Drug repositioning and repurposing: Terminology and definitions in literature. Drug Discov Today 20, 1027-1034.

[12] Travessa AM, Rodrigues FB, Mestre TA, Ferreira JJ (2017) Fifteen years of clinical trials in Huntington's disease: A very low clinical drug development success rate. J Huntingtons Dis 6, 157-163.

[13] Hay M, Thomas DW, Craighead JL, Economides C, Rosenthal J (2014) Clinical development success rates for investigational drugs. Nat Biotechnol 32, 40-51.

[14] DiMasi JA, Reichert JM, Feldman L, Malins A (2013) Clinical approval success rates for investigational cancer drugs. Clin Pharmacol Ther 94, 329-335.

[15] Rato ML, Duarte GS, Ferreira AN, Alves M, Mainoli B, Teodoro T, Mestre TA, Costa J, Ferreira JJ (2019) Nocebo response in Parkinson's disease: A systematic review and meta-analysis. Parkinsonism Relat Disord 65, 13-19.

[16] Food and Drug Administration. Amendments Act of 2007. Public Law 110-85. September 27, 2007. 
[17] Regulation (EU) No 536/2014 of the European Parliament and of the Council of 16 April 2014 on clinical trials on medicinal products for human use, and repealing Directive 2001/20/EC

[18] Decullier E, Chan A-W, Chapuis F (2009) Inadequate dissemination of phase I trials: A retrospective cohort study. PLoS Med 6, e1000034.

[19] De Angelis CD, Drazen JM, Frizelle FA, Haug C, Hoey J, Horton R, Kotzin S, Laine C, Marusic A, Overbeke AJ, Schroeder TV, Sox HC, Van Der Weyden MB (2005) Is this clinical trial fully registered?-A statement from the International Committee of Medical Journal Editors. $N$ Engl $J$ Med 352, 2436-2438.

[20] Paolini Paoletti F, Gaetani L, Parnetti L (2020) The challenge of disease-modifying therapies in Parkinson's disease: Role of CSF biomarkers. Biomolecules 10, 335.

[21] Dawson VL, Dawson TM (2019) Promising diseasemodifying therapies for Parkinson's disease. Sci Transl Med 11, eaba1659.
[22] Chen-Plotkin AS, Albin R, Alcalay R, Babcock D, Bajaj V, Bowman D, Buko A, Cedarbaum J, Chelsky D, Cookson MR, Dawson TM, Dewey R, Foroud T, Frasier M, German D, Gwinn K, Huang X, Kopil C, Kremer T, Lasch S, Marek K, Marto JA, Merchant K, Mollenhauer B, Naito A, Potashkin J, Reimer A, Rosenthal LS, Saunders-Pullman R, Scherzer CR, Sherer T, Singleton A, Sutherland M, Thiele I, van der Brug M, Van Keuren-Jensen K, Vaillancourt D, Walt D, West A, Zhang J (2018) Finding useful biomarkers for Parkinson's disease. Sci Transl Med 10, eaam6003.

[23] Cummings J, Lee G, Ritter A, Zhong K (2018) Alzheimer's disease drug development pipeline: 2018. Alzheimers Dement (N Y) 4, 195-214.

[24] Rodrigues FB, Duarte GS, Prescott D, Ferreira J, Costa J (2019) Deep brain stimulation for dystonia. Cochrane Database Syst Rev 1, CD012405.

[25] Groiss SJ, Wojtecki L, Südmeyer M, Schnitzler A (2009) Deep brain stimulation in Parkinson's disease. Ther $A d v$ Neurol Disord 2, 20-28. 